\title{
COMPRENSIÓN Y EXPLICACIÓN DEL DISCURSO PUBLICITARIO
}

\author{
Estrella Martínez-Rodrigo: Universidad de Málaga (España) \\ Rosario Segura García: Universidad de Granada (España)
}

\section{Introducción}

En las Investigaciones lógicas, Husserl (1859-1938) trata de hallar un método alternativo al psicologismo, el cual queda encerrado en el marco de un positivismo. En el momento en el que Husserl se plantea su investigación, el psicologismo consistía en hacerse una cierta composición de lugar llena de sentido común, con una apariencia de inteligibilidad inmediata.

Psicologismo ha habido siempre: Kant acusa de psicologismo a Hume y a los empiristas por estar demasiado pegados a la realidad. También en la antigua Grecia el empirismo era un problema para elaborar una teoría de conocimiento universal y ésta fue una de las dificultades fundamentales que los medievales veían en el planteamiento aristotélico, hasta que se realizó un estudio serio del mismo, por Avicena, Averroes y principalmente, Sto. Tomas de Aquino.

En las últimas décadas del siglo XIX, la psicología se había consolidado como ciencia, al probarse que era posible aplicar el método experimental para el estudio de la vida anímica de animales y seres humanos. Es el momento de W.Wundt, J.Mill, S.Spencer. Hay todo un afán de dotar de rigor a lo que se considera ciencia, y para eso, debe ser medible.

Es común considerar que tienen más rigor los datos estadísticos, medibles, que la profundización en contenidos. Esta postura puede estar presente en diversos planteamientos teóricos acerca de los estudios que se hacen de publicidad y comunicación.

Los que nos planteamos es que la comunicación audiovisual, también la publicitaria, es una ciencia social que participa de diversas dimensiones, no solo de la empírica. Se requiere también de una interpretación no sujeta sólo a estadísticas. La comunicación audiovisual y publicitaria es comunicación humana, que en algunos casos puede estar muy cerca de ser arte; en este último caso es mucho más difícil medir numéricamente el objeto, tratando de "explicar", una película o un spot publicitario. Los datos solo aportan una parte, en ocasiones, pequeña de la realidad. El sentido de algo, no viene de los datos.

Siguiendo a Dilthey, parece que hay cosas que se pueden explicar y otras comprender. La comunicación audiovisual requiere comprensión y explicación, y quizá necesite más de la primera que de la segunda, sin que esto signifique pierda rigor. Para entenderlo mejor acudiremos a reflexionar en la disputa filosófica 
acaecida en el siglo XIX acerca del método de las ciencias naturales, y las ciencias sociales, humanas o del espíritu.

\section{Comprensión y explicación: una disputa del siglo xix}

La importancia de la distinción de los métodos para alcanzar el entendimiento era de vital importancia tras la llustración. Históricamente las ciencias sociales se van separando poco a poco de la filosofía natural, y en consecuencia adquieren autonomía. Gran Bretaña y Francia impulsaron las nuevas ciencias sociales como una respuesta al nuevo orden social que vino después de la Revolución industrial y la Revolución francesa. El motivo fue estudiar y comprender las reglas que dirigían este nuevo orden.

Augusto Comte (1798-1857), fue quién, propuso que las ramas del conocimiento deberían limitarse al estudio de los hechos reales sin tratar de conocer sus causas primeras ni sus propósitos últimos, pues quería evitar caer en supuestos teológicos o metafísicos. Esta nueva forma de plantear el problema del conocimiento hizo crecer la creencia de que la realidad es "objetiva", en el sentido de medible, y por tanto deberíamos abordarla mediante los descubrimientos obtenidos empíricamente. También era general la creencia de que el sujeto que conoce debe ser neutral. Estamos ante el nacimiento del positivismo. Un término fundamental en este planteamiento era el de ley: ¿qué ley mueve la naturaleza? ¿Y la sociedad? ¿No sería una ley similar?

Por su parte, Wilhelm Dilthey (1833-1911) fue quien hizo una distinción sobre los objetivos epistémicos de las ciencias del siglo XIX, y criticó las ideas de los positivistas. Distinguió los métodos de conocimiento entre las ciencias naturales y ciencias humanas. Mientras que la tarea principal de las ciencias naturales es de llegar a explicaciones a base de leyes, la tarea básica de las ciencias humanas es el entendimiento de vida humana e histórica: "explicamos por procesos puramente intelectuales, pero comprendemos por la cooperación de todos los poderes de la mente activada por la aprehensión". (Dilthey, 1978: 172)

Es decir, las ciencias humanas tienen el objetivo de preguntarse por el significado de las acciones humanas, y el acceso a su conocimiento no es deductivo como una simple representación teórica mecanicista. Por el contrario, estas ciencias deben partir de la experiencia vivida, ya que esta está presente a nosotros en forma de valores relevantes a nuestros objetivos.

El tema de la comprensión y su metodología sería, en el siglo siguiente, tratado con una mayor profundidad por Max Weber. Para el positivismo de Comte, el conocimiento es considerado como algo que viene del exterior, que se puede reducir a explicaciones de carácter cuantitativo y causal, y podemos establecer las regularidades. Weber (1975: 67) sostiene la idea que una acción sólo puede ser interpretada porque está basada en una atribución radicalmente subjetiva de significado y el valor que uno tiene de ella.

El problema que se le plantea a esta postura es que el conocimiento queda limitado por la subjetividad y es irreductible a una explicación cuantitativa. 
Para entender mejor cada una de estas dos posturas aparentemente irreductibles, podemos resumir las premisas del método de cada una de ellas de la siguiente manera:

a) Método explicativo:

- El mundo real es objetivo y cognoscible

- Existen leyes naturales universales y el comportamiento social está relacionado a esas leyes.

- Se pueden distinguir las leyes jurídicas que normalizan los comportamientos humanos de las leyes naturales que rigen su comportamiento.

- Se pueden explicar las leyes causales que rigen la conducta de las sociedades.

b) Método comprensivo:

- La tarea principal de las ciencias humanas es el entendimiento de vida humana e histórica.

- Todos los sistemas intelectuales son cristalizaciones de una codificación mental de la experiencia que incluyen un modo particular organizado de la percepción cognoscitiva y responden a una situación compleja o espacio de estímulos más genérico arraigado en la vida.

- Estas ciencias deberían partir de la experiencia vivida ya que esta está presente a nosotros en forma de valores relevantes a nuestros objetivos.

\subsection{Historia del método explicactivo}

El origen del auge del método propio de las ciencias naturales hay que situarlo en relación a la Revolución Científica, y en hombres como Descartes, Bacon, Galileo, Newton, quienes aportaron las ideas básicas de lo que posteriormente se ha transformado en el enfoque científico que rige hasta el día de hoy.

Bacon reaccionó contra la lógica aristotélica, específicamente el silogismo, como instrumento para aprehender la realidad. Según él esa lógica no captaba la cosa y no era posible esperar de ella un avance del conocimiento. Su idea era buscar el conocimiento preguntándole directamente a la Naturaleza colocándola en una situación en que se viera forzada a suministrar respuestas: el experimento. Por otra parte, Bacon afirmaba que la tecnología sería la fuente de una nueva epistemología. La elevación de la tecnología al nivel de la filosofía y la postulación del concepto de experimento como una situación artificial que arrancara bajo apremio los secretos de la Naturaleza constituyeron los aportes principales de Bacon a lo que fue la revolución científica.

Descartes parte también criticando al Escolasticismo pero su preocupación principal era la certeza. Consideraba que la ciencia estaba basada sobre meras opiniones tomadas de la filosofía y comenzó por ponerlas todas en duda. Desprovisto el pensamiento de toda credulidad pudo abocarse a descubrir un método de pensamiento tan riguroso que asegurase certeza al ser aplicado a cualquier fenómeno que quisiese estudiar. Lo descubrió en las matemáticas y la geometría. Decía que la ciencia debía convertirse en una "matemática universal" puesto que los números eran la única prueba de certidumbre. 
La geometría, por su parte, aportaba el método para abordar los problemas: la división de ésta en sus unidades más simples, y una vez comprendidas y resueltas dichas unidades finalmente rearmar el problema completo, resuelto en cada uno de sus componentes. Un método simple y mecánico aplicable a cualquier objeto. Según Descartes, la mente del hombre poseyendo este método puede conocerlo todo.

La idea de que el hombre puede conocerlo todo por vía de su razón implicó para Descartes la separación entre mente y cuerpo y entre sujeto y objeto, ideas que se han transformado en pilares básicos del enfoque científico. Por otra parte, su método de conocimiento basado en la geometría en el que la división de un problema en sus partes y luego la recomposición en largas cadenas de raciocinios lo hizo reafirmar el principio aristotélico de la no contradicción, otro de los supuestos básicos del enfoque científico.

La unión de los aportes de Bacon y Descartes, el empirismo y el racionalismo, que en su época aparecieron como dos epistemologías opuestas, constituyen el más sólido fundamento de la revolución científica.

Los trabajos de Galileo primero y Newton después encarnaron esta mentalidad, revolucionaria para su época.

La revolución científica cambia radicalmente la conciencia y la vida del hombre. Lo hace valorizar la utilidad, el logro, lo práctico; lo hace creer sólo en lo visible, confiar sólo en su razón; lo hace olvidarse del ser en el hacer y el tener.

Así, desde el momento en que la ciencia natural hubo sentado sus bases intelectuales y los estudios humanísticos con pretensiones científicas alcanzaron a unirse a ella, resultó natural que una de las principales cuestiones de la metodología y de la filosofía de la ciencia del siglo XIX fuera lo concerniente a las relaciones entre estas dos importantes ramas de la investigación empírica. Las principales posiciones al respecto pueden engarzarse en las dos importantes tradiciones (aristotélica y galileana) del pensamiento metodológico que hemos distinguido. Como dice von Wright (1979):

"Una de estas posiciones es la filosofía de la ciencia típicamente representada por Auguste Comte y John Stuart Mill. Es la comúnmente llamada positivismo. El nombre, como ya se dijo, fue acuñado por Comte, pero usado con la debida cautela también es apropiado para caracterizar la posición de Mill y toda una tradición intelectual que partiendo de Comte y Mill no sólo desemboca en nuestros días, sino que se remonta hacia atrás hasta alcanzar a Hume y a la filosofía de la llustración".

Pero, ¿qué sostiene el positivismo? Tal vez, una forma en que podemos mejor comprenderlo es conociendo sus principios:

- El primer principio se refiere al monismo metodológico, o la idea de la unidad del método científico por entre la diversidad de objetos temáticos de la investigación científica

- El segundo principio es la consideración de que las ciencias naturales exactas, en particular la física matemática establecen un canon o ideal metodológico que mide el grado de desarrollo y perfección de todas las demás ciencias, incluidas las humanidades. 
- El tercer principio consiste en una visión característica de la explicación científica. Comte no ofrece una versión sistemática de la explicación. Su mayor énfasis recae sobre la predicción: el verdadero espíritu positivo consiste sobre todo en ver para prever, en investigar lo que es a fin de concluir de ello lo que será, conforme al dogma general de la invariabilidad de las leyes naturales.

Tal explicación es "causal", en un sentido amplio, tal como la concibe Mill el cual considera que un hecho individual queda explicado señalando su causa, esto es, estableciendo la ley o las leyes causales de las que su producción resulta instancia. Consiste, más específicamente, en la subsunción de casos individuales bajo leyes generales hipotéticas de la naturaleza la explicación de los hechos, y ya no es otra cosa en lo sucesivo que la relación establecida entre los distintos fenómenos particulares y ciertos hechos generales, incluida la naturaleza humana. Mill (1984) señala: que la ciencia de la naturaleza humana existe en la medida en que las verdades aproximadas que componen un conocimiento práctico del género humano, pueden revelarse corolarios de las leyes universales de la naturaleza humana en que se fundan.

La actitud hacia las explicaciones finalistas, por ejemplo, hacia los ensayos de dar razón de los hechos en términos de intenciones, fines, propósitos, conduce o bien a rechazarlas como acientíficas, o bien a mostrar que, una vez debidamente depuradas de restos "animistas" o "vitalistas", vienen a transformarse en explicaciones causales (von Wright, 1979).

En su aplicación a las ciencias sociales, en general, se admite que el positivismo implica dos postulados estrechamente vinculados. El primero es que los objetivos, los conceptos y los métodos de las ciencias naturales son aplicables a las indagaciones científico sociales. El segundo es la convicción de que el modelo de explicación utilizado en las ciencias naturales proporciona las normas lógicas en base a las cuales pueden valorarse las explicaciones dadas por las ciencias sociales.

\subsection{Hacia una comprensión en las ciencias}

La otra posición en el debate sobre las relaciones entre las ciencias de la naturaleza y las ciencias del hombre fue una reacción contra el positivismo. La filosofía antipositivista de la ciencia, que alcanza un lugar prominente a finales del siglo XIX, representa una tendencia mucho más diversificada y heterogénea que el positivismo.

Entre las figuras representativas de este tipo de pensamiento se incluyen eminentes filósofos, historiadores y científicos sociales alemanes. Entre ellos los más conocidos son Droysen, Simmel, Dilthey y Max Weber. Wierdelband y Rickert, de la escuela neokantiana de Baden, son afines a ellos. Del italiano Croce y del eminente filósofo de la historia y del arte, el británico Collingwood, puede decirse que pertenecen al ala idealista de esta tendencia anti positivista en metodología.

Todos estos pensadores rechazan el monismo metodológico del positivismo y rehúsan tomar el patrón establecido por las ciencias naturales exactas como ideal regulador, único y supremo, de la comprensión racional de la realidad.

Muchos de ellos acentúan el contraste entre las ciencias que, al modo de la física, la química o la fisiología, aspiran a generalizaciones sobre fenómenos reproducibles y 
las ciencias que, como la historia, buscan comprender las peculiaridades individuales y únicas de sus objetos.

Dilthey (1833-1911) rechaza la tendencia de fundar un conocimiento sobre lo humano siguiendo los procedimientos de las ciencias naturales.

Para Dilthey, la experiencia concreta y no la especulación representa el único punto de partida admisible para desarrollar lo que llama las ciencias del espíritu o del hombre (Geisteswissenschaften). El pensamiento no puede ir más allá de la vida, sostendrá... Dilthey es considerado el fundador de la corriente psicológica llamada descriptiva o de la comprensión. Ella se opone a la idea de una psicología explicativa (Echevarría, 1993: 99).

El uso común no hace una distinción aguda entre las palabras 'explicar' y 'comprender'. Cabe decir que prácticamente cualquier explicación, sea causal o teleológica o de otro tipo, nos proporciona una comprensión de las cosas. Pero 'comprensión' cuenta además con una resonancia psicológica de la que carece 'explicación'. Desde este carácter psicológico se considera la comprensión, como método característico de las humanidades, es una forma de empatía (Einfüblang) o recreación en la mente del estudioso de la atmósfera espiritual, pensamientos, sentimientos y motivos, de sus objetos de estudio.

Sin embargo, no es únicamente por este sesgo psicológico por lo que cabe diferenciar a la comprensión de la explicación. La comprensión se encuentra además vinculada con la intencionalidad de una manera en que la explicación no

lo está. Se comprenden los objetivos y propósitos de un agente, el significado de un signo o de un símbolo, el sentido de una institución social o de un rito religioso. Esta dimensión intencional o, como también seguramente podría decirse, esta dimensión semántica de la comprensión ha llegado a jugar un papel relevante en la discusión metodológica más reciente.

Sostiene von Wright que la noción diltheyana de comprensión era en un principio psicologista y subjetivista. Luego, al parecer, bajo la creciente influencia de Hegel, Dilthey acentúo el carácter objetivo de los frutos del método de comprensión. Y, Echeverría (1984: 99) agrega:

"Dilthey recibe una importante influencia de la escuela romántica, a través de autores como Goethe, Novalis y el propio Schleiermacher, que reivindicaban un retorno a la vida y un deseo por acceder a lo inmediato y a la totalidad. Simultáneamente con ello, sin embargo, Dilthey va a profesar el ideal desarrollado desde las ciencias naturales por alcanzar un conocimiento que sea o objetivamente válido."

El gran objetivo de Dilthey consiste, precisamente, en desarrollar una metodología apropiada para el entendimiento de las obras humanas, que eluda el reduccionismo y mecanicismo de las ciencias naturales. La vida debe ser entendida a partir de la propia experiencia de la vida. Las ciencias humanas no pueden pretender la comprensión de la vida a través de categorías externas a ella, sino a través de categorías intrínsecas, derivadas de ella misma. De manera gráfica Dilthey señala que "Por las venas del 'sujeto cognoscitivo' construido por Locke, Hume y Kant, no corre sangre verdadera"

Dilthey emprende dicha tarea entendiendo que se trata de un problema que no es metafísico, sino epistemológico; que requiere la profundización de nuestra 
conciencia histórica, y que requiere, por sobre todo, concentrarse en las expresiones (obras) que resultan de la propia vida. Dilthey adopta un enfoque más cercano a la fenomenología, ceñida a las experiencias concretas de los hombres.

Es central en la concepción planteada por Dilthey la distinción entre las ciencias naturales y las ciencias del espíritu. Mientras las primeras descansan en el concepto de fuerza propuesto por la física y en las matemáticas, las ciencias humanas se apoyan en el concepto de 'sentido' y en la historia. Los estudios sobre lo humano disponen de algo que está ausente en las ciencias naturales: la posibilidad de entender la experiencia interior de un otro a través de un misterioso proceso de transferencia mental. Dilthey, siguiendo a Schleiermacher (considerado padre de la hermenéutica moderna), concibe esta transposición como una reconstrucción de la experiencia interior del otro. Lo que interesa a Dilthey, sin embargo, no es el entendimiento de la otra persona, sino del mundo que a través de ello se revela.

El concepto clave en las ciencias del espíritu es el del entendimiento o la comprensión (Verstehen). Las ciencias naturales generan conocimiento a través de la explicación de la naturaleza; los estudios del hombre (las ciencias del espíritu) lo hacen a través de la comprensión de las expresiones de la vida. La comprensión permite acceder al conocimiento de la entidad individual; las ciencias naturales sólo se preocupan de lo individual como un medio para llegar a lo general, al tipo. Es más, las ciencias del espíritu, según Dilthey, son epistemológicamente anteriores a las de la naturaleza, a las que, por lo demás, abarcan pues toda ciencia natural es también un producto histórico.

La fórmula hermenéutica de Dilthey pone el énfasis en tres conceptos claves: la experiencia, la expresión y la comprensión o entendimiento.

a) El concepto de experiencia propuesto por Dilthey anticipa uno de los aspectos centrales de la filosofía posterior de Heidegger. No en vano este último reconoce el acierto de Dilthey. En efecto, la experiencia para Dilthey no es el contenido de un acto reflexivo de la conciencia. Es más bien el propio acto de la conciencia. No es algo que se halla fuera de conciencia y que ésta aprehende. La experiencia a la que alude Dilthey es algo mucho más fundamental, algo que existe antes de que el pensamiento reflexivo acometa la separación entre sujeto y objeto. Representa una experiencia vivida en su inmediatez, un ámbito previo al pensamiento reflexivo. Al distinguir de esta forma pensamiento y vida (experiencia), Dilthey coloca los cimientos a partir de los cuales se desarrollará la fenomenología en el siglo XX.

De lo anterior se deduce que representa un error considerar a la experiencia invocada por Dilthey como una realidad subjetiva. La experiencia aludida apunta a aquella realidad que se me presenta antes de convertirse en experiencia objetiva y, por lo tanto, antes de que lo subjetivo también se constituya. La experiencia representa un ámbito anterior, previo, a la separación sujeto-objeto, un ámbito en el cual el mundo y nuestra experiencia de él se hallan todavía unidos. En él tampoco se separan nuestras sensaciones y sentimientos del contexto total de las relaciones mantenidas juntas en la unidad de la experiencia.

b) El segundo término clave de la fórmula hermenéutica de Dilthey es el de la expresión. Por ella se entiende cualquier cosa que refleja la huella de la vida interior del hombre. Se trata de las 'objetivaciones' de la vida humana. Para Dilthey la hermenéutica debe concentrarse en estas expresiones objetivadas de la experiencia por cuanto le permiten al entendimiento dirigirse a elementos fijos, objetivos, y eludir 
así el intento de capturar la experiencia a través del esquivo procedimiento de la introspección. No olvidemos, por lo demás que Dilthey busca alcanzar un conocimiento objetivamente válido. La introspección es descartada por cuanto genera una intuición que no puede comunicarse, o bien, una conceptualización de ella que es, ella misma, una expresión objetivada de la vida interior.

Dilthey clasifica las distintas manifestaciones de la experiencia humana interior en: las manifestaciones de la vida (que incluye ideas y acciones) y las expresiones de la experiencia vivida. Estas últimas son para Dilthey las más importantes dado que la experiencia humana interior alcanza en ellas su más plena expresión. Dentro de ellas, el papel preponderante lo tienen las obras de arte, en la medida en que en ellas no sólo se manifiesta su autor, sino la vida misma, como sucede, por ejemplo, con las obras literarias. De allí que, para Dilthey, la hermenéutica no comprende sólo la teoría de la interpretación de los textos, sino de cómo la vida se manifiesta y expresa en obras.

c) El tercer término de la fórmula hermenéutica propuesta por Dilthey es el de la comprensión o el entendimiento (Verstehen). A la naturaleza, la explicamos; al hombre, señala Dilthey, lo comprendemos. Llevamos a cabo la explicación a través de procesos puramente intelectuales; pero para comprender es necesaria la actividad combinada de todos los poderes mentales de la aprehensión. La inteligencia, señala Dilthey, existe como realidad en los actos vitales de los hombres, todos los cuales poseen también los aspectos de la voluntad y de los sentimientos, por lo cual (la inteligencia) existe como realidad sólo dentro de la totalidad de la naturaleza humana. La comprensión no es, por lo tanto, sólo un acto del pensamiento; es la transposición y vuelta a experimentar el mundo tal como otra persona lo enfrenta en una experiencia de vida. Por lo tanto, la comprensión supone una transposición pre reflexiva de uno en un otro. Ello implica el redescubrimiento de uno en el otro.

Si se acepta una demarcación metodológica fundamental entre las ciencias naturales y las ciencias del espíritu o del hombre, surgirá inmediatamente la cuestión de dónde situar a las ciencias sociales y a las ciencias de la conducta. Estas ciencias nacieron en buena medida bajo la influencia de una presión cruzada de las tendencias positivistas y anti positivistas en el último siglo.

No es sorprendente por lo tanto el que hayan venido a resultar un campo de batalla para las dos tendencias en liza en la filosofía del método científico. La aplicación de métodos matemáticos a la economía política y a otras formas de estudio social fue un legado de la llustración del siglo XVIII que encontró apoyo en los positivistas del siglo XIX. El mismo Comte (como se ha repetido antes) acuñó el nombre de 'sociología' para el estudio científico de la sociedad humana. De los dos grandes sociólogos del cambio de siglo, Emile Durkheim fue esencialmente un positivista en todo lo referente a su metodología, mientras que en Max Weber se entremezclaba un cierto tinte positivista con el énfasis en la teleología y en la comprensión empática.

Entre las dos guerras mundiales resurgió el positivismo con más vigor que nunca. Este nuevo movimiento fue llamado neopositivismo o positivismo lógico, más tarde también se le conoció por empirismo lógico. El atributo 'lógico' fue añadido para indicar el apoyo que el redivivo positivismo obtuvo de los nuevos desarrollos en lógica formal. 
El resurgimiento de la lógica, después de medio milenio de decadencia y estancamiento (aproximadamente desde 1350 hasta 1850, con la excepción de las contribuciones de Leibniz en el siglo XVII) ha sido un evento de la mayor importancia en si mismo para la metodología y la filosofía de la ciencia.

En términos generales, los grandes intentos de superar la lógica tradicional los encontramos, en primer lugar, en el desarrollo de la dialéctica, iniciada por Hegel en su variante idealista $y$, posteriormente, propuesta por Marx, en su variante materialista y, en segundo lugar, en aquellos desarrollos que se iniciaran a partir del análisis lógico de los números efectuados por Frege y, posteriormente, continuados por Russel y otros.

La revolución lógica de Frege, en cambio, pondrá en duda el supuesto de que todas las proposiciones se someten a una estructura predictiva y, desde allí, introducirá algunas importantes distinciones correctivas relacionadas con el principio de identidad.

Sin embargo, difícilmente cabría decir que la lógica formal se halla comprometida intrínsecamente con el positivismo o con una filosofía positivista de la ciencia.

Por su parte, el positivismo lógico de los años 1920 y 1930 fue el principal, aun si no el único, afluente del que se nutrió la más amplia corriente de pensamiento filosófico hoy comúnmente conocida como filosofía analítica. Sería totalmente erróneo considerar que la filosofía analítica en su conjunto representa una rama del positivismo. Pero es acertado reconocer que las contribuciones de la filosofía analítica a la metodología y a la filosofía de la ciencia se han mantenido hasta hace bien poco predominantemente fieles al espíritu positivista, si por positivismo se entiende una filosofía partidaria del monismo metodológico, y de ideales matemáticos de perfección. sin embargo en los últimas décadas, ha habido un acercamiento de esta postura a entender lo que es el razonamiento práctico.

Los trabajos de Elizabeth Anscombe (1957), William Dray (1957), Melden (1961), Kenny (1963), d'Arcy (1963), Brown (1968) y otros reflejan el creciente interés, en el seno de la filosofía analítica, por el concepto de acción y por las formas del discurso práctico. Pero no fue hasta la aparición del importante trabajo de Charles Taylor en 1964, cuando esta nueva orientación de la filosofía analítica llegó a conectar con la teoría de la explicación en psicología y en las otras ciencias de la conducta.

\section{Cuando analizamos un discurso publicitario, ¿qué tipo de objeto estamos analizando?}

3.1. Publicidad

Podemos hacer nuestra la definición de F. Atanasio (1976: 17):

"Llamamos, por tanto, publicidad a toda forma remunerada de presentación o de promoción, llevada a cabo generalmente por parte de terceras personas, de un producto o servicio por cuenta de un destinatario identificado, efectuada con el fin de inducir a los compradores potenciales o a aquellos que tienen influencia sobre las compras a considerar favorablemente el producto o servicio, o a asumir una actitud positiva en relación con el mismo." 
Lo primero que destaca en la definición es que es una forma de comunicación que tiene la finalidad de persuadir. La publicidad coincide con la retórica en la utilización del lenguaje y, sobre todo, en una finalidad común: la persuasión.

De modo resumido podemos decir que el objetivo de la publicidad es persuadir al receptor con un mensaje para que tome la decisión de compra de un producto o servicio que una empresa ofrece. En el caso de la publicidad institucional, seria persuadirle a generar en él una actitud favorable a una entidad o a los proyectos que promocionen dicha entidad. La persuasión del discurso publicitario se consigue a través de la utilización de un lenguaje connotativo y denotativo y de una combinación de lenguaje verbal y no verbal.

Es decir, el discurso publicitario es una forma de comunicación humana en el que la persuasión ejerce un papel primordial.

Si nos propusiéramos hacer ciencia sobre este discurso, qué método deberíamos elegir ¿un método explicativo, o comprensivo? Pensamos que principalmente requiere un método comprensivo y la aplicación de un método explicativo, es un añadido que puede enriquecer el análisis, facilitarlo en cuanto herramienta, pero no ser el fundamento de dicho análisis porque el objeto del que estamos hablando no se rige exactamente por causas y efectos, ni está sujeto a leyes de las ciencias experimentales sino se trata de un objeto propio del espíritu humano y una muestra de ello es que se ponen en juego las emociones, y la inteligencia, algo difícil de medir o explicar, y más fácil de comprender.

\subsection{Emociones, persuasión y publicidad}

La persuasión publicitaria podría quedar caracterizada como la presentación o promoción de un producto por medio de terceros con la intención de provocar en el posible comprador la consideración positiva sobre dicho producto. A lo largo de la historia, la publicidad ha ido evolucionando desde la presentación directa del objeto, hasta su aparición indirecta e incluso disimulada. Se juega con la imaginación, con la memoria y con las emociones del posible comprador. Es sabido que un mensaje por radio apela especialmente a la imaginación y que un mensaje por televisión aporta datos visuales además de sonoros, que completan la percepción. Pero el desarrollo de las tecnologías permite que cada vez sea más sofisticado este juego: el anuncio audiovisual ha ido evolucionando hacia la creación de mini historias, en las que el producto se camufla y es cada vez más difícil de descubrir, lo que provoca la curiosidad y la búsqueda por parte del espectador; es decir, se impele al interés. Es lo que ha quedado resumido en el modelo publicitario AIDA: atención, interés, deseo, adquisición.

El producto es disimulado ocultando sus valores reales, creando una ficción, erotizándolo, o siendo presentado por alguien ajeno a la propia empresa que cuenta su experiencia positiva por el uso; es el caso de los testimoniales. Pero queremos llamar la atención sobre el hecho de que, ante todo, se observa en la retórica publicitaria un incremento de la estrategia de apelar a los afectos y emociones de una forma cada vez más intensa. Este recurso puede dificultar con frecuencia la racionalidad, el uso de la reflexión del espectador que podría dejar al descubierto dicha estrategia; son guiños que contribuyen a crear el deseo y la pasión por adquirir lo anunciado. 
Como señalan Tellis y Redondo (2000: 229), la publicidad tiene capacidad de suscitar emociones y persuadir de una manera eficaz. Frente a las emociones, un discurso lógico o racional presenta ventajas e inconvenientes, lo que lleva a considerar que es necesario realizar un buen uso de ellas en la técnica publicitaria para lograr el objetivo propuesto: convencer al espectador de la calidad, de la utilidad o de la belleza del producto e incitar a su adquisición. Las ventajas del recurso a las emociones en publicidad se sitúan en la línea de requerir menos esfuerzo en la percepción del mensaje, enriquecerlo afectivamente y facilitar que se recuerde mejor; hay una mayor facilidad para una respuesta rápida, y por tanto, disminuye una posible reacción defensiva.

Por otro lado, entre los inconvenientes que presenta su utilización podrían enumerarse los siguientes: pueden distraer respecto al contenido del mensaje; si las emociones suscitadas son desagradables (miedo, asco), predisponen a no ver el anuncio; si hay desproporción entre la emoción y el mensaje, se puede provocar que el público sospeche que hay instrumentalización de sus sentimientos y no se logra la respuesta adecuada. Algunos publicistas pueden incluso hacer uso de esta desproporción de modo intencionado para llamar la atención sobre el producto; realizan una estrategia de transgresión, y aunque es criticada por una parte del público, pretenden introducir dicha crítica en su estrategia publicitaria, al hablarse más de la marca o producto. Como ejemplo podría citarse algunos anuncios de la marca Bennetton.

El modo como se pueden suscitar emociones es diverso: representaciones, historias, demostraciones, recursos al humor o a la música. Quizá el modo más eficaz sea el de las representaciones, pues apelan directamente a que el público emplee su lógica y genere sus emociones; es decir, se provoca una identificación de sus sentimientos con los del protagonista del anuncio. En el resto de los modos, se da cierta distancia entre el espectador y el anuncio, y la identificación es menor. Aun así, parece que el modo más frecuente de persuasión es aquel que apela solo a las emociones y provoca apasionar.

Los motivos pueden ser variados, y no es infrecuente querer impedir la actuación de un discurso lógico que tal vez haga descubrir cierta manipulación. En estos casos más que de persuasión cabría hablar de seducción. También las emociones que se suscitan en el ámbito publicitario son muy variadas y dependen del mensaje que se quiera transmitir: miedo, ternura, risa, nobleza. Hay una emoción que destaca sobre todas ellas a la hora de hora de hacer su aparición en la dinámica de la publicidad: es el amor romántico; de ahí que una socióloga israelí, Eva Illouz centre su análisis de la relación emoción-publicidad precisamente en el amor romántico.

Para ser capaces de captar la dinámica de esta relación interpersonal se hace más necesario una comprensión a modo de una hermenéutica de sentido, que una explicación de causas y efectos (explicación).

\section{Conclusión}

Las conclusiones que extraemos de nuestro trabajo son las siguientes: 
- El análisis de objetos publicitarios o audiovisuales se realiza de un modo más completo y profundo si se aborda desde una perspectiva en los que se combinan elementos estadísticos con otros como antropológicos, históricos, filosóficos, etc.

- Este tipo de análisis abre las puertas a nuevas consideraciones acerca de la sociedad y el hombre que pueden influir notablemente en el modo de hacer publicidad.

- Comúnmente, aparecen recursos en la publicidad que no son técnicos, sino humanos, su mejor o peor uso requiere una comprensión de los mismos que no puede limitarse a una definición sino a una tradición cultural o histórica, en este sentido, una comprensión de esos recursos se hace necesaria.

- Aunque parezca que se escapa del ámbito publicitario, llama la atención como el debate intelectual puede haberse distanciado y mucho de la vida social real, no la que se pretende construir desde instituciones políticas o del estado. La publicidad puede ser un modo de percibirlo. por su propia naturaleza, las campañas publicitarias desean ser eficaces y mover los resortes reales del espectador, ya sea por motivos loables como evitar los accidentes de tráfico, o menos loables como aumentar las ganancias, no importa ganar un debate sino ganar en la realidad. Es decir, tomando la expresión diltheyana, el publicista busca "la sangre verdadera".

- El status de una posible teoría de la comunicación audiovisual y/publicitaria atraviesa por estas reflexiones que nos llevan a considerar que se trataría de una ciencia social, en la que conviven comprensión y explicación con predominio de la primera, sin quedar atrapada en datos técnicos 0 estadísticas que reducirían el conocimiento de su potencial persuasivo y estético.

\section{Referencias}

ATtANASIO, F. (1967). Publicidad hoy Métodos y técnicas, Deusto: Bilbao.

COMTE, A. (1989). Discurso sobre el espíritu positivo, Alianza Editorial: Madrid.

DILTHEY, W. (1978). Introducción a las ciencias del espíritu, Ed. FCE: México.

ECHEVERRÍA, R. (1993). El Búho de Minerva, Dolen Ediciones: Santiago de Chile.

HABERMAS, J. (1999). Teoría de la acción comunicativa, Taurus: Madrid.

HUSSERL, Edmund (1976). Investigaciones Lógica, Revista de Occidente: Madrid.

MILL, J.S: (1984). El utilitarismo: El sistema de la lógica, Alianza Editorial: Madrid.

TELLIS, G. y REDONDO, I. (2000). Estrategias de publicidad y promoción. Pearson Education: Madrid.

VON WRIGHT, G.H.(1979). Explicación y comprensión, Alianza Editorial: Madrid. 
WEBER, M. (1975). La "objetividad" del conocimiento en la ciencia social y en la política social, Alianza Editorial: Madrid. 